\title{
Improving Anytime Behavior for Traffic Signal Control Optimization Based on NSGA-II and Local Search
}

\author{
Phuong Thi Mai Nguyen \\ De Montfort University's \\ Interdisciplinary Research Group in \\ Intelligent Transport Systems (DIGITS) \\ and Centre for Computational \\ Intelligence (CCI)
}

De Montfort University, Leicester, UK

Email: phuongthimainguyen@gmail.com

\author{
Benjamin N. Passow \\ De Montfort University's \\ Interdisciplinary Research Group in \\ Intelligent Transport Systems (DIGITS) \\ and Centre for Computational \\ Intelligence (CCI) \\ De Montfort University, Leicester, UK \\ Email: benpassow@ieee.org
}

\author{
Yingjie Yang \\ De Montfort University's \\ Interdisciplinary Research Group in \\ Intelligent Transport Systems (DIGITS) \\ and Centre for Computational \\ Intelligence $(\mathrm{CCI})$
}

De Montfort University, Leicester, UK

Email: yyang@dmu.ac.uk

\begin{abstract}
Multi-Objective Evolutionary Algorithms (MOEAs) and transport simulators have been widely utilized to optimise traffic signal timings with multiple objectives. However, traffic simulations require much processing time and need to be called repeatedly in iterations of MOEAs. As a result, traffic signal timing optimisation process is time-consuming. Anytime behaviour of an algorithm indicates its ability to return as good solutions as possible at any time during its implementation. Therefore, anytime behavior is desirable in traffic signal timing optimisation algorithms. In this study, we propose an optimisation strategy (NSGA-II-LS) to improve anytime behaviour based on NSGAII and local search. To evaluate the validity of the proposed algorithm, the NSGA-II-LS, NSGA-II and MODEA are used to optimize signal durations of an intersection in Andrea Costa scenario. Results of the experiment show that the optimization method proposed in this study has good anytime behaviour in the traffic signal timings optimization problem.
\end{abstract}

\section{INTRODUCTION}

Traffic congestion in urban areas has been becoming a serious problem which can lead to an increase in fuel consumption, air pollution and detrimental impacts on economic growth. Traffic signal control is a cost effective tool to ease congestion in urban traffic network. It is estimated that 50$80 \%$ of traffic incidents happens at intersections and theirs surroundings, $1 / 3$ travel time and $80-90 \%$ waiting time is consumed at red phases of signalized intersections [1]. Therefore, properly and efficiently operation of traffic signal control of the overall traffic network is critical to the performance of the whole traffic system.

The role of traffic signal timing optimization is to significantly improve network performance by optimizing objectives such as reducing delay, number of stops and increasing network throughput or average speed in the network. Setting traffic signals in a signal-controlled street network involves the determination of cycle time, splits of green (and red) time, and offsets. Traffic light signal optimization might optimize a part of or all these values. Traffic signal timing optimization consists of two main categories: mathematical programming method and simulation-based approach [2].

The former scheme utilizes mathematical formulations to capture the characteristics of traffic flow model which will be utilized to optimize objectives in traffic management. However, the interrelationship between the flows of complex intersections can not be adequately captured by mathematical programming formulations [2]. For that reason, more recently, researchers tend to optimize traffic signal timing by using simulation-based approaches ( [2], [3], [4], [5]). According to level of detail which transport simulators can represent, they are divided into three categories: microscopic, macroscopic and mesoscopic traffic simulators. Macroscopic simulators describe the traffic at a high level of aggregation without considering its parts. Microscopic traffic models simulate the movement of individual vehicles. Mesoscopic models are at an intermediate level of detail, for example, describing the individual vehicle but not their interactions. Traffic characteristics achieved from traffic models are used to generate a near-optimal set of signal timing plans via the optimization algorithms. However, it is noted that traffic simulation using these models takes a significant amount of processing time.

Evolutionary Algorithms (EAs) are widely used to solve the multi-objective optimisation problem in transportation ([6], [7], [8], [9], [10], [11]). However, in a traffic signal plan optimization process, traffic simulation needs to be called every time an individual is evaluated. Time to run multiple simulations as a part of the evolutionary algorithm requires much processing time. The computation time will rapidly rise as the scale of the traffic network increases, such as road network size and number of simulated vehicles. Moreover, when applying EAs to optimise traffic signal timings, the optimization process is time-consuming since EAs need to run the scenario simulation many times [12]. [13] utilizes parallel genetic algorithm reduce time response of the optimization process while [14] combined GA with cluster computing. 
[15] introduced GPU based NSGA-II to reduce computation burden.

The optimization literature is mostly focused on the quality of solutions reached by an algorithm after a given time. However, such studies might not work efficiently in optimization problems where function evaluations are limited by time or cost. In these situations, in order to evaluate efficiency of an optimisation algorithm, an indicator, which can measure ability of that algorithm to produce good solutions at any time during its operation, is need. Anytime behavior of an algorithm is its ability to provide as good solutions as possible at any time during its execution and continuously improves the quality of the results as computation time increases ([16], [17]).

Anytime behavior may be described in terms of the curve of hypervolume over time. Hypervolume, introduced by Zitzler and Thiele [18], measures the volume of the objective space which is dominated by a non-dominated set. Therefore, if one non-dominated set has a higher hypervolume, it will be closer to Pareto-optimal front. Hypervolume indicator is used to compare anytime behavior between two multi-objective optimization algorithms. Because optimizing traffic signal control is time-consuming and the time to run the optimization process is limited and scenario specific, therefore, anytime behavior of the system is desirable.

In this work we propose a multi-objective optimization strategy for a traffic light control system. The proposed algorithm (NSGA-II-LS ) which is based on NSGA-II and a local search has better anytime behavior comparing to NSGA-II and MODEA.

\section{RELATED WORK}

\section{A. Traffic light signals timing problem}

General form of a multi-objective optimization problem in traffic light signal timing can be described as follows:

$$
\begin{array}{rr}
\min / \max & f_{m}(x), m \in(1, M) \\
\text { s.t } & C_{\min }<C<C_{\max } \\
& x_{k}^{L}<x_{k}<x_{k}^{U}
\end{array}
$$

where $M$ is the number of the objectives functions, $C$ is the cycle length, $C_{\min }$ and $C_{\max }$ are minimum and maximum values of the cycle length.$x\left(x_{1}, x_{2}, \ldots, x_{n}\right)$ is a vector composed of $\mathrm{n}$ decision variables $x_{i}, i \in(1, n) . x_{k}^{L}$ and $x_{k}^{U}$ are lower and upper bound of variable $x_{i}$. Objectives functions in transportation management would be average delay ([2],[19]) and queue lengths ([20],[21]) at signalized intersections, average travel time ([3], [14]), travel cost [22], flow ([2], [14],[21]) or traffic exhaust emissions ([1], [23]). $f_{m}(x)$ are functions of offset time, cycle time, green time of phases or order of the phases in the intersection.

\section{B. NSGA-II and SUMO}

Elitist Non-dominated Sorting Genetic Algorithm (NSGAII) is a well established multi-objective optimization algorithm and is an instance of an Evolutionary Algorithm which was proposed by K. Deb and his students in 2000. Deb et al.

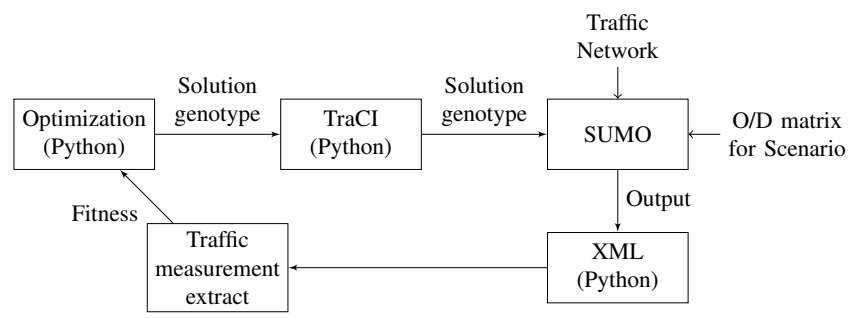

Fig. 1: Overview of simulation setup for the network.

pointed out that NSGA-II, in most problems, is able to find much better spread of solutions and better convergence near the true Pareto-optimal front compared to Pareto-archived evolution strategy and strength-Pareto EA.

SUMO is a open source traffic simulator which can be used for various purposes such as the evaluation of changes in infrastructure and policy before implementing them on the road [25]. SUMO has been used to simulate traffic in a number of studies ([26], [27], [28], [29] and [30]). SUMO is a microscopic traffic simulation so it can describe every vehicles in detail such as departure and arrival time, lanes to use, velocity and positions. Therefore, it is very suitable for evaluating traffic light strategies. Furthermore, SUMO is able to to generate a large number of different measures. Values of all these measurement data will be collected into files or a socket connection following the common rules for writing files. All output files generated by SUMO are in XMLformat [25]. All needed traffic measurements can be extracted by using any programming language. In this work, python interfaces including TraCI [25] has been used (Figure 1).

\section{METHODOLOGY}

\section{A. The Proposed Local Search Strategy}

Local search is a metaheuristic method which can be used for solving hard optimization problems. A local search algorithm starts from a candidate solution and then iteratively moves to a neighbor solution in the search space until a local optimum is found. In this study, a local search scheme is introduced to the evolutionary optimiser NSGA-II. After solutions are non-dominated sorted into fronts, the local search is applied for these fronts. The local searching strategy given in the following.

Given a front $F_{t}$ with size of $N P$ with $i^{t h}$ solution $x_{i, t}\left(x_{1, i, t}, x_{2, i, t}, \ldots, x_{n, i, t}\right)$ in $F_{t}$ where $n$ is the number of variables, $t$ is numerical order of the front. The selected neighborhoods discovering strategy is based on [31]. There are two neighborhoods on the $k^{t h}$ variable of a solution $x_{i, t}$ are defined in [31]:

$$
\begin{aligned}
& n b 1_{k, i, t}=x_{k, i, t}+c *\left(u_{k, i, t}-v_{k, i, t}\right) \\
& n b 1_{k, i, t}=x_{k, i, t}-c *\left(u_{k, i, t}-v_{k, i, t}\right)
\end{aligned}
$$

where $u_{i, t}$ and $v_{i, t}$ are two solutions in the same front with $x_{i, t}$. Therefore $2 * n$ neighborhoods are explored on a solutions. However, in our proposed method, in order to reduce the 
number of solution evaluations, only two neighbours of each individual are discovered. Each variables in two neighbours $n b 1_{i, t}$ and $n b 2_{i, t}$ of $x_{i, t}$ are calculated by using (2) and (3).

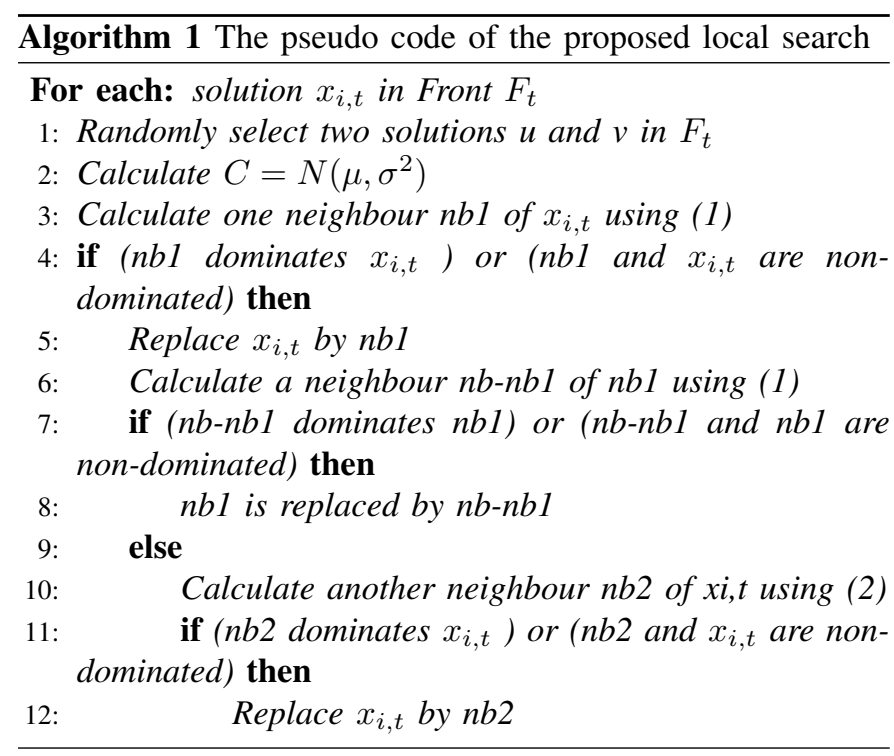

In order to decide whether the neighbour solution $n b$ can replace the current solution $x$, replacement strategy is given as follows: if the neighbour solution dominates the current solution or they are non-dominated, the neighbour solution will replace the current neighbour. It is well known that replacing the current solution $x$ with one neighbourhood which dominate $x$ would help the current solution move closer to the Pareto optimal front. In case the current solution $x$ and its neighbour solution $n b$ are non-dominated, the replacement of $x$ by $n b$ will help to increase the diversity of solutions.

During the searching process, if the first seeking direction (using (2) to create neighbour) gives a worse result comparing with the current solution, the searching scheme will turn into another direction by using (3). As a result, the searching procedure would have more chance to achieve a better solution. A new population of size $N P$ are created by performing the local search on all fronts. All solutions in the new population are as good as or better than the individuals in the current population.

\section{B. The Proposed Algorithm NSGA-II-LS}

The basic idea of the proposed system is the combination of NSGA-II and the local search. The pseudo code of the local search is described in algorithm 1. The framework of the proposed algorithm is illustrated in the algorithm 2. The input to the approach is an initial set of $N$ random individuals. This population undergoes a number of generations until the termination criteria satisfy. In each iteration, tournament selection, recombination and mutation operators are used to created offspring population of size $N$. First, a combined population of size $2 N$ is formed from parent and children population. Then, the population is sorted according to nondomination. $N$ best solutions are selected using their ranking and crowed-comparison operator. Suppose that these $N$ best solutions belong to set $F$ including $K$ fronts. Local search is applied with each front in $F$ to find out new solutions which are as good as or better than the current solutions in that front (line 9). The new aggregate population of size $N$ from $K$ fronts after local search procedures is now used for selection, crossover, and mutation to create a new population in the next generation.

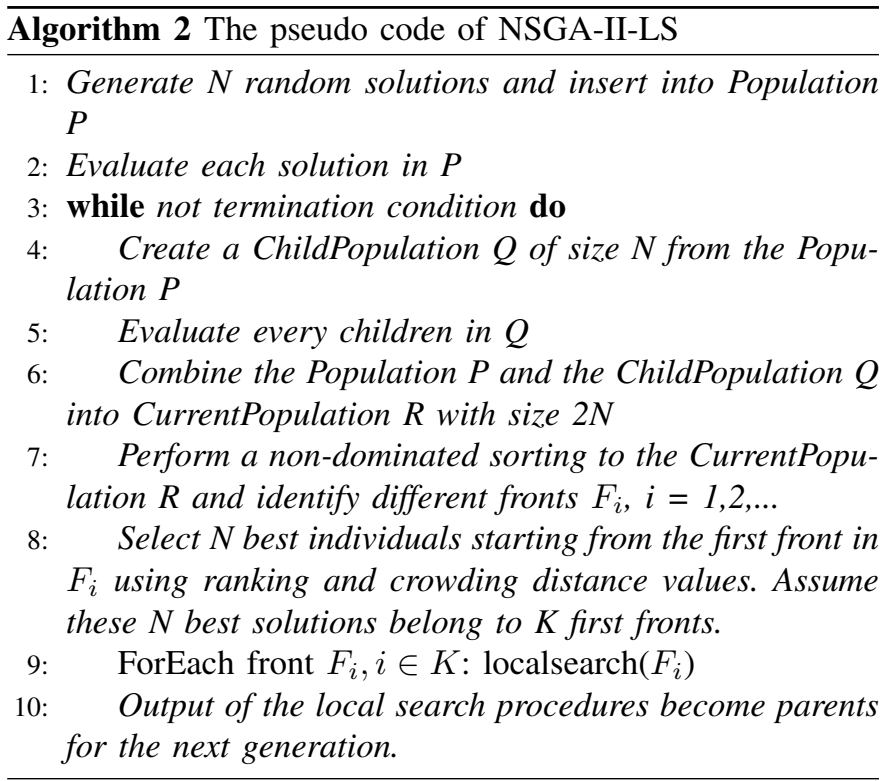

Each generation in NSGA-II includes $N$ evaluations (line 5) to calculate fitness values with the population size of $N$. Therefore, there are total $N * M$ number of evaluations where $M$ is the number of generation in NSGA-II. Local search procedure (algorithm 1) includes 2 evaluations for 2 neighbours of each solution in the front. Consequently, $2 * N$ number of evaluations need to be performed in the local search procedure in each iteration. Offspring solutions also need to be estimated. Therefore, there are $3 * N$ and $3 * N * M$ number of solution evaluations in each generation and in the whole program of the proposed algorithm, respectively.

\section{EXPERIMENTAL RESULTS}

\section{A. Experimetal Scenario}

To study the performance of the introduced and discussed optimisation algorithms in traffic light timing control, a real world traffic scenario from the city of Bologna was chosen [32]. In this experiment, Andrea Costa scenario (Figure 2(a)) simulated the traffic conditions of the area around the football stadium in big events such as football matches or concerts is chosen. The scenario consists of traffic demand for Andrea Costa's peak hour from 8:00am to 9:00am. This scenario includes positions and traffic light plan of all traffic lights in the traffic network. A traffic light control program (id=210) of coordinated intersections (Figure 2(b)) in the Andrea Costa scenario will be optimized using the proposed algorithm.

The traffic lights which include their positions and signal plans are provided so the aim in this experiment is to optimize 


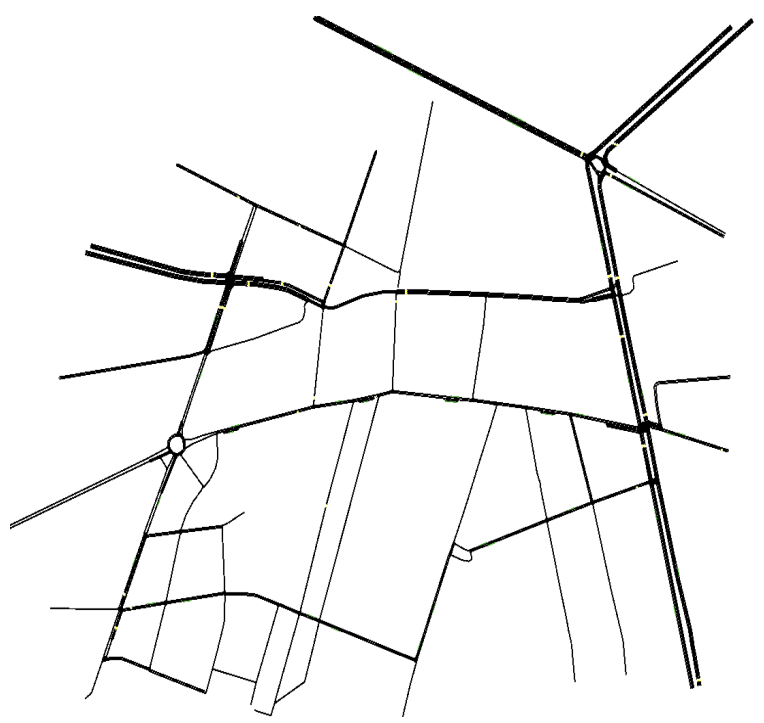

(a) Andrea Costa Scenario

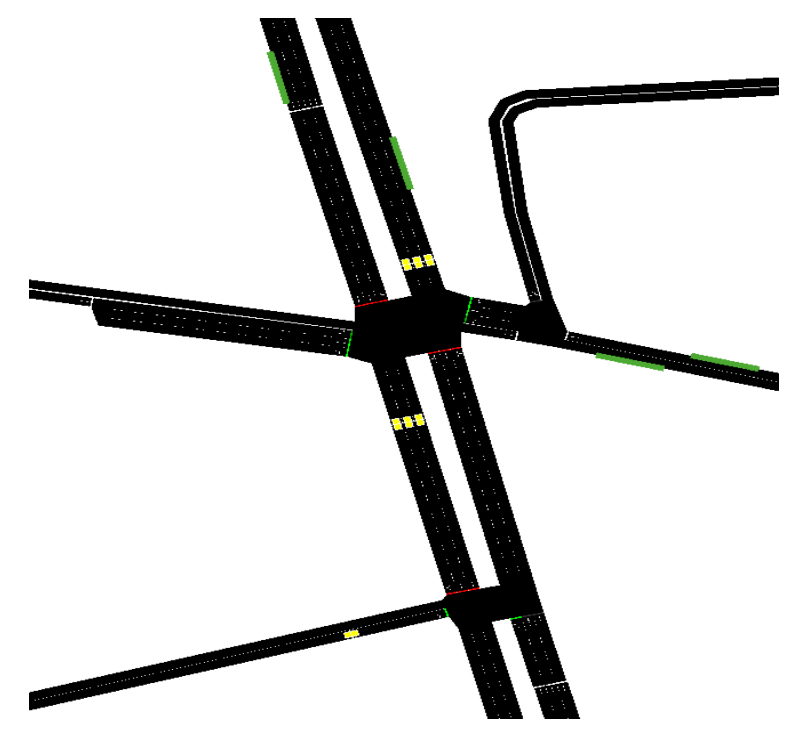

(b) Intersection id $=210$

Fig. 2: Scenario of the experiment.

phase durations of the signal plan of the traffic light. The proposed algorithm optimizes two objectives concurrently which are minimize total time loss of whole Andrea Costa traffic network and maximize the number of vehicles which enter and left the simulated scenario.

The cycle in the traffic signal strategy consists of 17 phases. Therefore, an individual in the optimization process is represented as $x=\left(x_{1}, x_{2}, \ldots x_{17}\right)$ where $x_{i}, i \in[1,17]$ has a integer value in range from 1 to 80 . The constrain in this study is that the total length of all phases in a cycle must be in range from 80 to 250 . If one individual is infeasible, its fitness value will be add a penalty value to reduce its ability to be chosen to be parents. The proposed algorithm NSGA-II-LS is compared with NSGA-II and MODEA in term of hypervolume and diversity indicators.

\section{B. Parameters for optimisation algorithms}

We conducted two different experiments that use same traffic scenario described in the previous section. The first experiment compares NSGA-II-LS with NSGA-II while the second experiment evaluates the performances of NSGA-II-LS versus MODEA. In order to compare the anytime behavior of optimisation algorithms, both experiments were run 20 times. While in each separate run, the two compared algorithms were started from the same initial random population, each separate run of the 20 repeats had a separate initial randomly generated population. This allowed for better comparison, as the initial population acted as a reference point to calculate the hypervolume indicators in every runs, being the same for both the experiments.

1) Optimisation parameters for NSGA-II: Parameters for NSGA-II algorithm are listed in Table I. Crossover scheme utilized in our study is Simulated Binary Crossover (SBX) with $\eta^{c}=20$. A mutated variable with probability of $1 / 17$
TABLE I: Optimisation parameters for NSGA-II

\begin{tabular}{|c|c|}
\hline Parameters & Values \\
\hline Population size & 20 \\
\hline Number of generations & 45 \\
\hline Number of variables & 17 \\
\hline Number of evaluations & $20 * 45+20=920$ \\
\hline Mutation rate of a solution & 1.0 \\
\hline Mutation rate of a variable in an individual & $1.0 / 17$ \\
\hline Crossover rate & 1.0 \\
\hline
\end{tabular}

using Polynomial Mutation scheme. An individual in NSGAII has 17 variables which are durations of 17 phases of the cycle of the traffic signal control program in the intersection $\mathrm{id}=210$. Reference point is $\mathrm{R}(1.1,1.1)$.

2) Optimisation parameters for NSGA-II-LS: As discussed in previous parts, NSGA-II-LS is a combination of NSGAII and the proposed local search. Therefore, optimisation parameters of NSGA-II-LS consist of parameters of NSGA-II which is mentioned above and parameters of the local search. In the proposed local search which was illustrated in algorithm 1 , parameter $c$ is calculated by a normal distribution with $\mu=0$ and $\sigma=3$. The iteration number of the algorithm is 15 generations with 20 solutions in the population.

3) Optimisation parameters for MODEA: In our study, candidate creation strategy utilized in MODEA is DE/rand/1/bin scheme. The differential weight/scaling factor $F=0.8$ and the crossover probability $R=0.9$. Population size of MODEA is 20 individuals and the algorithm runs in 45 generations.

\section{Performance measures}

For evaluating the efficiency of NSGA-II-LS, NSGA-II and MODEA, mean values of hypervolume in 20 runs are calculated. Moreover, other related statistics measurements such as standard deviation, median, min and max values of the hypervolume have been used. 
Hypervolume of NGSA-II-LS and NSGA-II

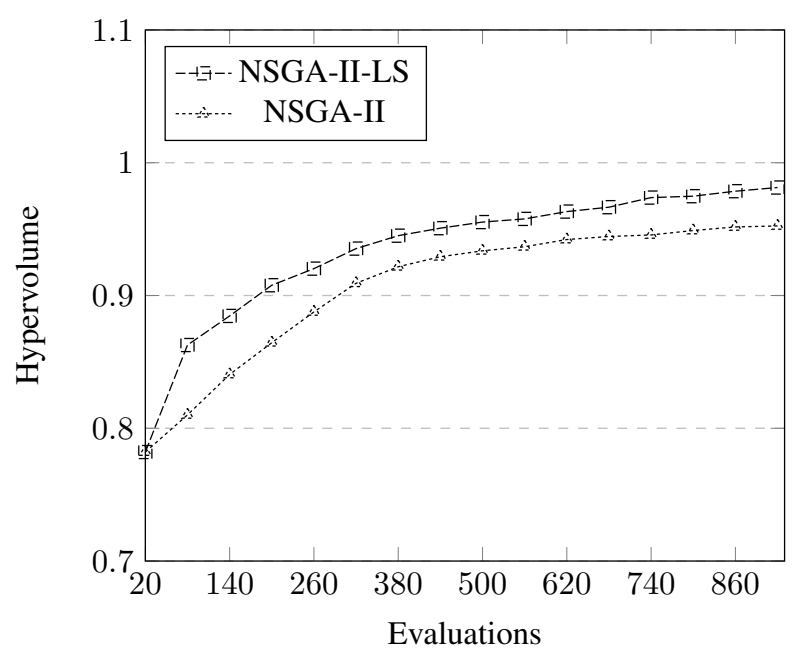

(a) Comparing NSGA-II-LS and NSGA-II using hypervolume

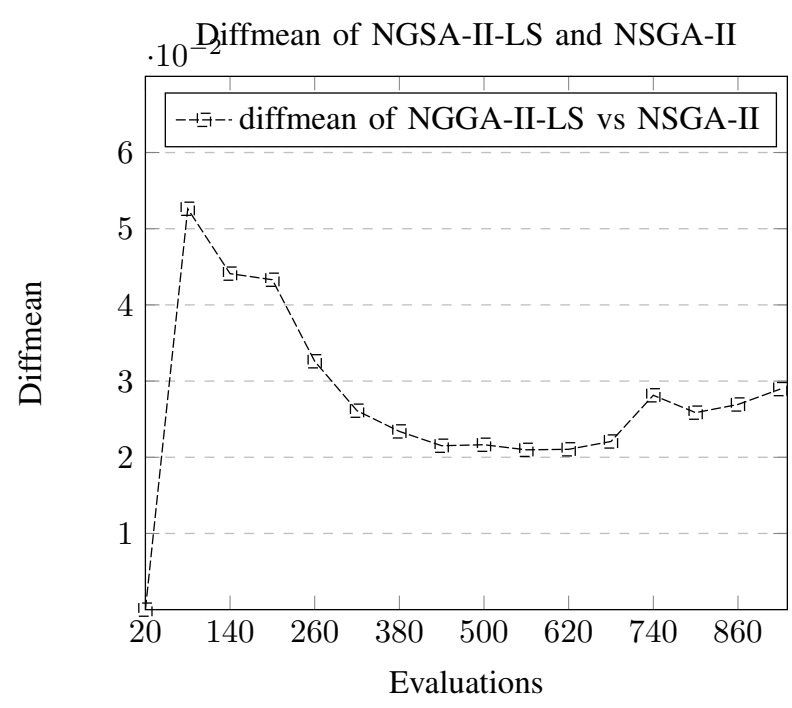

(b) Comparing NSGA-II-LS and NSGA-II using Diffmean

Fig. 3: Comparision between NSGA-II-LS and NSGA-II.

\begin{tabular}{|c|c|c|c|c|c|}
\hline Evaluation & Mean & stdDev & Median & Min & Max \\
\hline 20 & 0.78211 & 0.01774 & 0.778 & 0.7575 & 0.82404 \\
\hline 80 & 0.81007 & 0.01867 & 0.811435 & 0.77114 & 0.84561 \\
\hline 140 & 0.840509 & 0.01894 & 0.837995 & 0.80787 & 0.87617 \\
\hline 200 & 0.864435 & 0.02398 & 0.86644 & 0.82191 & 0.91703 \\
\hline 260 & 0.887627 & 0.02368 & 0.8808 & 0.85444 & 0.93302 \\
\hline 320 & 0.909207 & 0.02471 & 0.904385 & 0.86818 & 0.94545 \\
\hline 380 & 0.921655 & 0.02094 & 0.92103 & 0.8809 & 0.95096 \\
\hline 440 & 0.929193 & 0.02059 & 0.93436 & 0.88408 & 0.95494 \\
\hline 500 & 0.93366 & 0.02031 & 0.9418 & 0.88934 & 0.96076 \\
\hline 560 & 0.936715 & 0.01942 & 0.942775 & 0.89207 & 0.96383 \\
\hline 620 & 0.942159 & 0.02121 & 0.94598 & 0.89233 & 0.97885 \\
\hline 680 & 0.944445 & 0.02174 & 0.94884 & 0.89233 & 0.979 \\
\hline 740 & 0.945616 & 0.0206 & 0.949505 & 0.89433 & 0.97946 \\
\hline 800 & 0.948943 & 0.01584 & 0.94984 & 0.91195 & 0.97946 \\
\hline 860 & 0.951636 & 0.01684 & 0.94987 & 0.91868 & 0.98882 \\
\hline 920 & 0.952385 & 0.01657 & 0.949965 & 0.92001 & 0.98935 \\
\hline
\end{tabular}

(a) Results of NSGA-II

\begin{tabular}{|c|c|c|c|c|c|}
\hline Evaluation & Mean & stdDev & Median & Min & Max \\
\hline 20 & 0.78211 & 0.017743 & 0.778 & 0.7575 & 0.82404 \\
\hline 80 & 0.862689 & 0.041966 & 0.858565 & 0.793 & 0.93616 \\
\hline 140 & 0.884613 & 0.040351 & 0.874965 & 0.83247 & 0.96311 \\
\hline 200 & 0.907739 & 0.036938 & 0.90393 & 0.84021 & 0.97167 \\
\hline 260 & 0.920226 & 0.031643 & 0.925505 & 0.87536 & 0.97165 \\
\hline 320 & 0.93532 & 0.020136 & 0.93774 & 0.89876 & 0.97318 \\
\hline 380 & 0.945051 & 0.020524 & 0.94728 & 0.89975 & 0.98809 \\
\hline 440 & 0.950707 & 0.019395 & 0.951645 & 0.90437 & 0.98884 \\
\hline 500 & 0.955306 & 0.017141 & 0.95719 & 0.91486 & 0.99051 \\
\hline 560 & 0.957692 & 0.016752 & 0.959945 & 0.9171 & 0.99054 \\
\hline 620 & 0.963205 & 0.016615 & 0.966055 & 0.91964 & 0.99062 \\
\hline 680 & 0.966518 & 0.015434 & 0.968175 & 0.92445 & 0.99137 \\
\hline 740 & 0.973752 & 0.015613 & 0.971485 & 0.94813 & 1.00134 \\
\hline 800 & 0.974801 & 0.015402 & 0.97052 & 0.9533 & 1.00181 \\
\hline 860 & 0.978567 & 0.014211 & 0.974915 & 0.96061 & 1.00279 \\
\hline 920 & 0.981334 & 0.0151 & 0.976285 & 0.96297 & 1.01599 \\
\hline
\end{tabular}

(b) Results of NSGA-II-LS

Fig. 4: Statistics results of NSGA-II-LS and NSGA-II.

In addition, we define a metric to evaluate how the difference between mean values of two compared algorithms change. This metric is diffmean and described as follows:

$$
\text { diffmean }=\sum_{i=1}^{n}\left(\text { mean }_{A, i}-\text { mean }_{B, i}\right)
$$

where $\mathrm{A}$ and $\mathrm{B}$ are two compared algorithms, $n$ is the number of evaluations. mean $n_{A, i}$ and mean $_{B, i}$ are mean values of A and $\mathrm{B}$ at $i^{\text {th }}$ evaluation.

Furthermore, to evaluate the diversity of the proposed algorithm, two diversity indicators are used to compare the spread of the solutions found between NSGA-II-LS, NSGA-II and MODEA [33]. The first diversity performance measures is spacing metric of Schott $(S)$ which measures how evenly the points of approximated Pareto front are distributed in the objective space. Spacing is calculated as:

$$
S=\sqrt{\frac{1}{N-1} \sum_{m=1}^{N}\left(d_{a v g}-d_{m}\right)^{2}}
$$

with $d_{m}=\min _{j=1, \ldots, N} \sum_{k=1}^{M}\left|f_{k m}(x)-f_{k j}(x)\right|$ where $N$ is the number of the solutions in the found Pareto front and $M$ is the number of objective functions. $d_{\text {avg }}$ is the average value of all $d_{m}$ values. The smaller $S$, the more evenly distributed the solutions.

However, $S$ does not provide any information with regards to the extent of the solutions. Therefore, maximum spread 


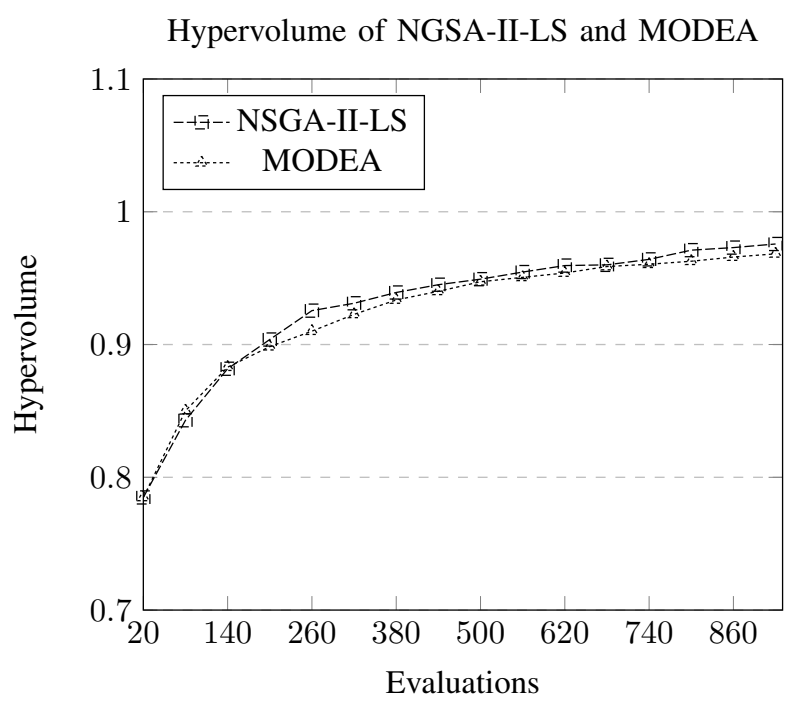

(a) Comparing NSGA-II-LS and MODEA using hypervolume

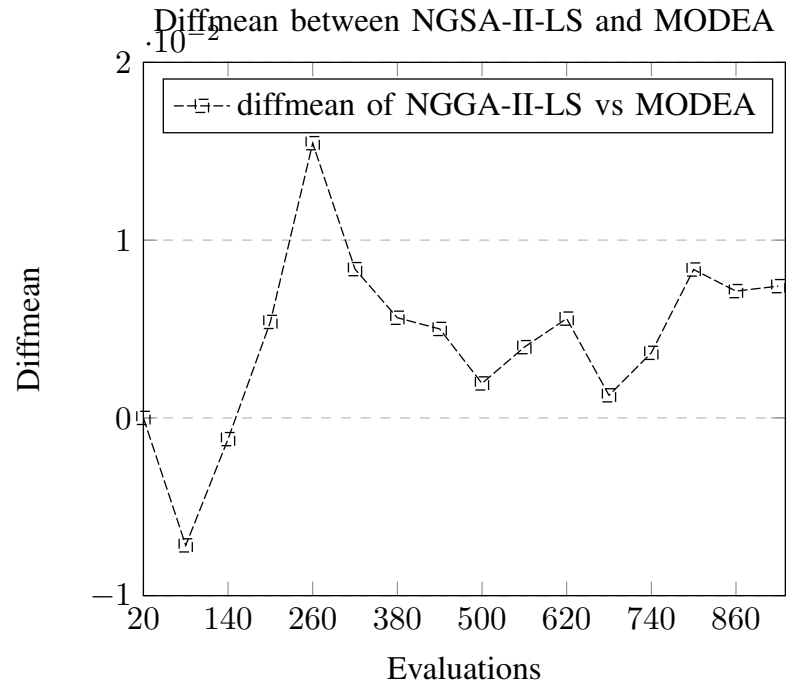

(b) Diffmean between NSGA-II-LS and MODEA

Fig. 5: Comparison between NSGA-II-LS and MODEA.

\begin{tabular}{|c|c|c|c|c|c|}
\hline Evaluation & Mean & stdDev & Median & Min & Max \\
\hline 20 & 0.784841 & 0.012184 & 0.78797 & 0.76532 & 0.80425 \\
\hline 80 & 0.850131 & 0.012534 & 0.854185 & 0.82047 & 0.86911 \\
\hline 140 & 0.8829515 & 0.017913 & 0.886565 & 0.85487 & 0.9095 \\
\hline 200 & 0.8983875 & 0.014418 & 0.898535 & 0.87204 & 0.92492 \\
\hline 260 & 0.910071 & 0.017266 & 0.906575 & 0.88453 & 0.951 \\
\hline 320 & 0.9227165 & 0.016434 & 0.92542 & 0.89287 & 0.95137 \\
\hline 380 & 0.9335635 & 0.016694 & 0.937755 & 0.90877 & 0.95488 \\
\hline 440 & 0.9400625 & 0.018031 & 0.94595 & 0.90944 & 0.96591 \\
\hline 500 & 0.9472865 & 0.015764 & 0.948645 & 0.91108 & 0.96865 \\
\hline 560 & 0.950607 & 0.014635 & 0.95379 & 0.91574 & 0.9687 \\
\hline 620 & 0.95388 & 0.014518 & 0.95565 & 0.91837 & 0.97643 \\
\hline 680 & 0.958744 & 0.014119 & 0.962775 & 0.92576 & 0.97938 \\
\hline 740 & 0.9604765 & 0.013387 & 0.96298 & 0.92651 & 0.97992 \\
\hline 800 & 0.962737 & 0.011767 & 0.963855 & 0.92831 & 0.98042 \\
\hline 860 & 0.96588 & 0.010813 & 0.96698 & 0.94042 & 0.98064 \\
\hline 920 & 0.968313 & 0.010861 & 0.96823 & 0.9448 & 0.9834 \\
\hline
\end{tabular}

(a) Results of MODEA

\begin{tabular}{|c|c|c|c|c|c|}
\hline Evaluation & Mean & stdDev & Median & Min & Max \\
\hline 20 & 0.784841 & 0.012184 & 0.78797 & 0.76532 & 0.80425 \\
\hline 80 & 0.8429605 & 0.031812 & 0.834485 & 0.79682 & 0.92371 \\
\hline 140 & 0.881759 & 0.039373 & 0.866375 & 0.8345 & 0.95967 \\
\hline 200 & 0.9037855 & 0.030173 & 0.897845 & 0.866 & 0.96478 \\
\hline 260 & 0.925525 & 0.033721 & 0.93256 & 0.87289 & 0.98867 \\
\hline 320 & 0.931084 & 0.027660 & 0.93901 & 0.88559 & 0.96738 \\
\hline 380 & 0.939198 & 0.020757 & 0.943425 & 0.90526 & 0.96813 \\
\hline 440 & 0.9450655 & 0.018799 & 0.94587 & 0.91222 & 0.9712 \\
\hline 500 & 0.949226 & 0.017828 & 0.954555 & 0.91632 & 0.9704 \\
\hline 560 & 0.954587 & 0.016656 & 0.962105 & 0.91633 & 0.97467 \\
\hline 620 & 0.9594655 & 0.015986 & 0.96353 & 0.92936 & 0.98051 \\
\hline 680 & 0.9600145 & 0.018554 & 0.96592 & 0.90597 & 0.98345 \\
\hline 740 & 0.964136 & 0.014379 & 0.96628 & 0.92518 & 0.98345 \\
\hline 800 & 0.9710835 & 0.016948 & 0.968385 & 0.94932 & 1.03212 \\
\hline 860 & 0.973011 & 0.016964 & 0.972225 & 0.94176 & 1.03234 \\
\hline 920 & 0.975725 & 0.015786 & 0.973695 & 0.95473 & 1.03235 \\
\hline
\end{tabular}

(b) Results of NSGA-II-LS

Fig. 6: Statistics results of NSGA-II-LS and MODEA.

measurement is utilized as the second diversity indicator.

$$
M S=\sqrt{\sum_{k=1}^{N}\left(\max _{k}-\min _{k}\right)^{2}}
$$

where $\max _{k}$ and $\min _{k}$ are maximum and minimum values of the $k^{t h}$ objective, respectively. $M S$ measures the length of the diagonal of the hyperbox that is created by the extreme values of the non-dominated set. The bigger $M S$, the more widely spread the solutions.

\section{Results}

1) NSGA-II-LS vs. NSGA-II: We compare our proposed algorithm NSGA-II-LS with NSGA-II using hypervolume and diversity indicators. In our experimental, we run these algorithms 20 times and hypervolume values are recorded. Figure 3(a) illustrates the mean values of hypervolume of NSGAII-LS and NSGA-II in 20 runs. The figure points out that with the same initial population, NSGA-II-LS achieves better hypervolume than NSGA-II in the same number of solution evaluations. Differences between mean values of NSGA-II-LS and NSGA-II are shown in Figure 3(b). The diffmean value soars at the beginning of the experiment (after 80 evaluations). It then decrease gradually and remain quite stable from $500^{t h}$ evaluation until the end of the optimisation process. Therefore, NSGA-II-LS achieves better anytime behavior as compared to NSGA-II. Figure 4 illustrates mean, standard deviation, median, min value and max values of hypervolume of both the algorithms in 20 runs, respectively.

Diversity performance of the proposed systems and NSGAII are presented in the table II. NSGA-II has smaller $S$ value than NSGA-II-LS which indicates that the solutions in the 
TABLE II: Diversity comparison between NSGA-II and NSGA-II-LS.

\begin{tabular}{|c|c|c|}
\hline Diversity performance measure & NSGA-II & NSGA-II-LS \\
\hline$S$ & $1,618,604.503$ & $7,733,930.815$ \\
\hline$M S$ & $5,364,304.997$ & $22,632595.04$ \\
\hline
\end{tabular}

TABLE III: Diversity comparison between MODEA and NSGA-II-LS.

\begin{tabular}{|c|c|c|}
\hline Diversity performance measure & MODEA & NSGA-II-LS \\
\hline$S$ & $5,643,745.306$ & $5,031,615.396$ \\
\hline$M S$ & $23,295,753.73$ & $24,218,440.85$ \\
\hline
\end{tabular}

non-dominated front of NSGA-II are more evenly distributed than these of NSGA-II-LS. However, $M S$ value of NSGAII-LS is much more higher than that of NSGA-II. Therefore, the non-dominated solutions found in the proposed algorithm spread more widely than these in NSGA-II.

2) MODEA vs. NSGA-II-LS: We repeated the optimisation process with MODEA and NSGA-II-LS algorithms 20 times. Figure 5(a) illustrates mean values of hypervolume in 20 runs of MODEA and NSGA-II-LS. We can see from the figure that the curves of hypervolume of MODEA and NSGA-IILS are very close. The difference between mean values of NSGA-II-LS and MODEA is presented in Figure 5(b). The diffmean value got negative values from 80 to 140 evaluations and positive values in the rest. It rises very quickly and peaks at 260 evaluations and then fluctuate during the rest of the simulations. We can see from the figure that the diffmean value is above "zero" line from $140^{t h}$ solution to the end of the optimisation process. Figure 6 presents statistics data in terms of hypervolume of MODEA versus NSGA-II-LS in 20 runs. It is conclude that in overall hypervolume of MODEA is slightly higher than that of NSGA-II-LS.

Table III illustrates the diversity measures of MODEA and NSGA-II-LS. Data in the table show that the proposed algorithm has better diversity performance in terms of both maximum spread and spacing between non-dominated solutions.

\section{CONClusion}

In real-time traffic signal optimization with expensive objective functions, anytime behaviour is desirable. An algorithm, which has anytime behavior, is able to return good solutions at any running time. In this paper we proposed a new multiobjective optimization algorithm based on NSGA-II and local search. The local search introduced in this study are integrated into iterations of NSGA-II. The local search would produce as good as or better solutions comparing with current solutions in the same fronts. Output of the local search procedure will become parents of the next generation. The experiment was conducted to compare our scheme with NSGA-II and MODEA in term of hypervolume indicator. The results show that our proposed algorithm is better than NSGA-II and as good as MODEA. Consequently, our proposed algorithm can return good solutions at an early stage of the optimization process. This is important in traffic management since urban traffic networks are highly dynamic and optimization processes are time-consuming. Therefore, better anytime behavior of the proposed system would help decision makers get good solutions without waiting until the optimisation process finish.

In future, traffic characteristics in over-saturated conditions need to be get involved in the optimisation process. Furthermore, we will study not only MOEAs but also other optimisation approaches. These will be studied and tested in regards to reduced response time of the optimisation process in the highly complex and dynamic urban transport environments. New optimisation methods need to be introduced to solve expensive multi-objective optimisation problems.

\section{REFERENCES}

[1] Z. Ben, S. Lei, and C. Dan, "Traffic intersection signalplanning multi-object optimization based on genetic algorithm," in Intelligent Systems and Applications (ISA), 2010 2nd International Workshop on, May 2010, pp. 1-4.

[2] Y.-Y. Chen and G.-L. Chang, "A macroscopic signal optimization model for arterials under heavy mixed traffic flows," Intelligent Transportation Systems, IEEE Transactions on, vol. 15, no. 2, pp. 805-817, April 2014.

[3] R. Abushehab, B. Abdalhaq, and B. Sartawi, "Genetic vs. particle swarm optimization techniques for traffic light signals timing," in Computer Science and Information Technology (CSIT), 2014 6th International Conference on, March 2014, pp. 27-35.

[4] J. Chen and L. Xu, "Road-junction traffic signal timing optimization by an adaptive particle swarm algorithm," in Control, Automation, Robotics and Vision, 2006. ICARCV '06. 9th International Conference on, Dec 2006, pp. 1-7.

[5] D. Jia and Z. Chen, "Traffic signal control optimization based on fuzzy neural network," in Measurement, Information and Control (MIC), 2012 International Conference on, vol. 2, May 2012, pp. 1015-1018.

[6] P. Li, "Scheduling and implementation of network product logistics supply chain under the evolutionary algorithm," in Intelligent Transportation, Big Data and Smart City (ICITBS), 2015 International Conference on, Dec 2015, pp. 701-704.

[7] Y.-J. Zheng, M.-X. Zhang, H.-F. Ling, and S.-Y. Chen, "Emergency railway transportation planning using a hyper-heuristic approach," Intelligent Transportation Systems, IEEE Transactions on, vol. 16, no. 1, pp. 321-329, Feb 2015.

[8] E.-G. Talbi, "Tutorial iii: Solving complex optimization problems," in Innovations in Information Technology (IIT), 2015 11th International Conference on, Nov 2015, pp. XXXVIII-XXXIX.

[9] A. S. C. F. E. D. G. M. P. B. N. . Y. Y. Goodyer, E., "Computational intelligence and its role in enhancing sustainable transport systems," International Journal for Traffic and Transport Engineering (IJTTE), vol. 1, pp. 180-186, 2013. 
[10] S. Witheridge, B. Passow, and J. Shell, "Logan's run: Lane optimisation using genetic algorithms based on nsga-ii," in Neural Networks (IJCNN), 2014 International Joint Conference on, July 2014, pp. 63-68.

[11] F. Caraffini, F. Neri, B. N. Passow, and G. Iacca, "Re-sampled inheritance search: high performance despite the simplicity," Soft Computing, vol. 17, no. 12, pp. 2235-2256, 2013. [Online]. Available: http://dx.doi.org/10.1007/s00500-013-1106-7

[12] Z. Shen, K. Wang, and F. Zhu, "Agent-based traffic simulation and traffic signal timing optimization with gpu," in Intelligent Transportation Systems (ITSC), 2011 14th International IEEE Conference on, Oct 2011, pp. $145-150$.

[13] Z. Guangwei, G. Albert, and L. D. Sherr, "Optimization of adaptive transit signal priority using parallel genetic algorithm," Tsinghua Science and Technology, vol. 12, no. 2, pp. 131-140, April 2007.

[14] J. Sanchez-Medina, M. Galan-Moreno, and E. RubioRoyo, "Traffic signal optimization in "la almozara" district in saragossa under congestion conditions, using genetic algorithms, traffic microsimulation, and cluster computing," Intelligent Transportation Systems, IEEE Transactions on, vol. 11, no. 1, pp. 132-141, March 2010.

[15] Z. Shen, K. Wang, and F.-Y. Wang, "Gpu based non-dominated sorting genetic algorithm-ii for multiobjective traffic light signaling optimization with agent based modeling," in Intelligent Transportation Systems - (ITSC), 2013 16th International IEEE Conference on, Oct 2013, pp. 1840-1845.

[16] M. Lpez-Ibez and T. Sttzle, "Automatically improving the anytime behaviour of optimisation algorithms," European Journal of Operational Research, vol. 235, no. 3, pp. 569 - 582, 2014.

[17] J. Dubois-Lacoste, M. Lpez-Ibez, and T. Sttzle, "Anytime pareto local search," European Journal of Operational Research, vol. 243, no. 2, pp. 369 - 385, 2015.

[18] E. Zitzler and L. Thiele, "Multiobjective optimization using evolutionary algorithms - a comparative case study," in Proceedings of the 5th International Conference on Parallel Problem Solving from Nature, ser. PPSN V. London, UK, UK: SpringerVerlag, 1998, pp. 292-304. [Online]. Available: http://dl.acm.org/citation.cfm?id=645824.668610

[19] S. Feng and Y. Xiaoguang, "Optimization algorithm of urban road traffic signal plan based on nsgaii," in Intelligent Computation Technology and Automation (ICICTA), 2008 International Conference on, vol. 2, Oct 2008, pp. 398-401.

[20] B. Yin, M. Dridi, and A. El Moudni, "Forward search algorithm based on dynamic programming for real-time adaptive traffic signal control," Intelligent Transport Systems, IET, vol. 9, no. 7, pp. 754-764, 2015.

[21] S. T. Yan Li, Lijie Yu and K. Chen, "Multi-objective optimization of traffic signal timing for oversaturated intersection," Mathematical Problems in Engineering, 2013.

[22] Y. Chin, K. Yong, N. Bolong, S. Yang, and K. Teo, "Multiple intersections traffic signal timing optimization with genetic algorithm," in Control System, Computing and Engineering (ICCSCE), 2011 IEEE International Conference on, Nov 2011, pp. 454-459.

[23] S. Zhou, X. Yan, and C. Wu, "Optimization model for traffic signal control with environmental objectives," in Natural Computation, 2008. ICNC '08. Fourth International Conference on, vol. 6, Oct 2008, pp. 530-534.

[24] K. Deb, A. Pratap, S. Agarwal, and T. Meyarivan, "A fast and elitist multiobjective genetic algorithm: Nsga-ii," Evolutionary Computation, IEEE Transactions on, vol. 6, no. 2, pp. 182-197, Apr 2002.

[25] C. P. D.Krajzewicz, G.Hertkorn. Sumo homepage. http://sumo.sourceforge.net.

[26] SUMO2014 - Modeling Mobility with Open Data, vol. 24. DLR-Institute of Transportation Systems, May 2014.

[27] SUMO 2015-Intermodal Simulation for Intermodal Transport, May 2015.

[28] M. B. Daniel Krajzewicz, Jakob Erdmann and L. Bieker, "Recent development and applications of sumosimulation of urban mobility," International Journal on Advances in Systems and Measurements, vol. 5, 2012.

[29] L. Bedogni, M. Gramaglia, A. Vesco, M. Fiore, J. Harri, and F. Ferrero, "The bologna ringway dataset: Improving road network conversion in sumo and validating urban mobility via navigation services," Vehicular Technology, IEEE Transactions on, vol. 64, no. 12, pp. 5464-5476, Dec 2015.

[30] P. Bedi, V. Jindal, R. Garg, and H. Dhankani, "A preemptive approach to reduce average queue length in vanets," in Advances in Computing, Communications and Informatics (ICACCI), 2015 International Conference on, Aug 2015, pp. 2089-2095.

[31] B. Chen, W. Zeng, Y. Lin, and D. Zhang, "A new local search-based multiobjective optimization algorithm," Evolutionary Computation, IEEE Transactions on, vol. 19, no. 1, pp. 50-73, Feb 2015.

[32] L. Bieker, D. Krajzewicz, A. Morra, C. Michelacci, and F. Cartolano, Modeling Mobility with Open Data: 2nd SUMO Conference 2014 Berlin, Germany, May 15-16, 2014. Cham: Springer International Publishing, 2015, ch. Traffic Simulation for All: A Real World Traffic Scenario from the City of Bologna, pp. 47-60.

[33] M. Helbig and A. P. Engelbrecht, "Performance measures for dynamic multi-objective optimisation algorithms," Information Sciences, vol. 250, pp. 61 - 81, 2013. 